\title{
Spinal cord stimulation: a nonopioid alternative for chronic pain management
}

\author{
Aaron Hong MD MSc, Vishal Varshney MD, Gregory M.T. Hare MD PhD, C. David Mazer MD
}

Cite as: CMAJ 2020 October 19;192:E1264-7. doi: 10.1503/cmaj.200229

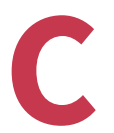

hronic pain affects 1 in 5 Canadians and is associated with considerable socioeconomic burden. ${ }^{1}$ Although opioids have been the mainstay of treatment, they have lost favourability owing to crises of addiction, abuse, tolerance and dependence. ${ }^{1,2}$ Consequently, alternatives - including cognitive behavioural therapy, physical rehabilitation, non-opiate pharmacology and integrative therapies - have been developed. ${ }^{1,3}$

When conventional therapies produce unacceptable adverse effects or do not provide sufficient pain relief, spinal cord stimulation (neuromodulation) may offer a rescue option, either alone or in conjunction with other modalities. ${ }^{3,4}$

Neuromodulation, defined as the alteration of nerve activity through targeted stimulus delivery, was first introduced in $1967.2,3,5 \mathrm{It}$ is based on the principle of electrically stimulating the dorsal column of the spinal cord, to mask pain signals. ${ }^{2}$ The technology has since advanced, and it was a Canadian neurosurgeon who helped demonstrate both the efficacy and cost-effectiveness of spinal cord stimulation in chronic pain treatment. ${ }^{6,7}$

Usage of spinal cord stimulators has increased worldwide, and according to the United States Food and Drug Administration, an estimated 50000 devices are implanted annually in the US (150 cases/million population; www.fda.gov/medical-devices/letters -health-care-providers/conduct-trial-stimulation-period-implanting -spinal-cord-stimulator-scs-letter-health-care-providers). However, uptake in Canada has been substantially lower, with 172 implantations performed in Ontario in 2018 (12 cases/ million population). ${ }^{3}$ This difference is likely related, at least in part, to a lack of awareness about spinal cord stimulation and high upfront device costs $(\$ 20000-\$ 30000) .^{3}$

\section{What is spinal cord stimulation?}

Spinal cord stimulation consists of leads placed in the epidural space alongside the dorsal column and then tunnelled subcutaneously to an implantable pulse generator. These generators contain a battery and microprocessor (similar to a pacemaker) that are internalized within a subcutaneous pocket either within the abdominal wall or posteriorly in the flank or gluteal region, and programmed transcutaneously while allowing remote control by the physician or patient. Implantable pulse generators

\section{KEY POINTS}

- Spinal cord stimulation masks pain signals through a transcutaneous implantable electric pulse generator.

- Spinal cord stimulation is safe, efficacious and cost-effective in chronic pain management of neuropathic pain conditions, including failed back surgery syndrome, chronic regional pain syndrome and chronic peripheral neuropathies.

- Newer spinal cord stimulation technologies are expanding clinical indications such as visceral and ischemic pain, with potential for further improved efficacy.

- Increased awareness of and access to spinal cord stimulation therapy may allow more Canadians to benefit from relief of intractable chronic pain and may reduce opioid consumption.

last 5-10 years, depending on rechargeability, and are replaceable. Patients are asked not to twist, bend or stretch excessively for 6-8 weeks postoperatively. After this period, patients may resume most physical activities.

The generators continuously emit low-frequency electrical pulses (tonic stimulation) that mask pain signals travelling up the spinal cord and replace them with nonpainful paresthesias. ${ }^{8}$ Wall and Melzack's gate control theory is the most accepted proposed pain relief mechanism, which asserts that nonpainful inputs close the "nerve-gates" to painful inputs, thereby preventing pain sensations from reaching the brain ${ }^{2,7}$ (Figure 1).

\section{How is treatment delivered?}

Initially, spinal cord stimulators were implanted by spine surgeons, necessitating general anesthesia, laminectomy, electrode placement directly onto dura, and postoperative hospital admission. Over the last decade, development of percutaneous epidural leads inserted under fluoroscopic guidance has enabled specially trained anesthesiologists or other pain specialists to safely implant spinal cord stimulators as outpatient procedures, using light sedation and local anesthesia (Figure 2). 


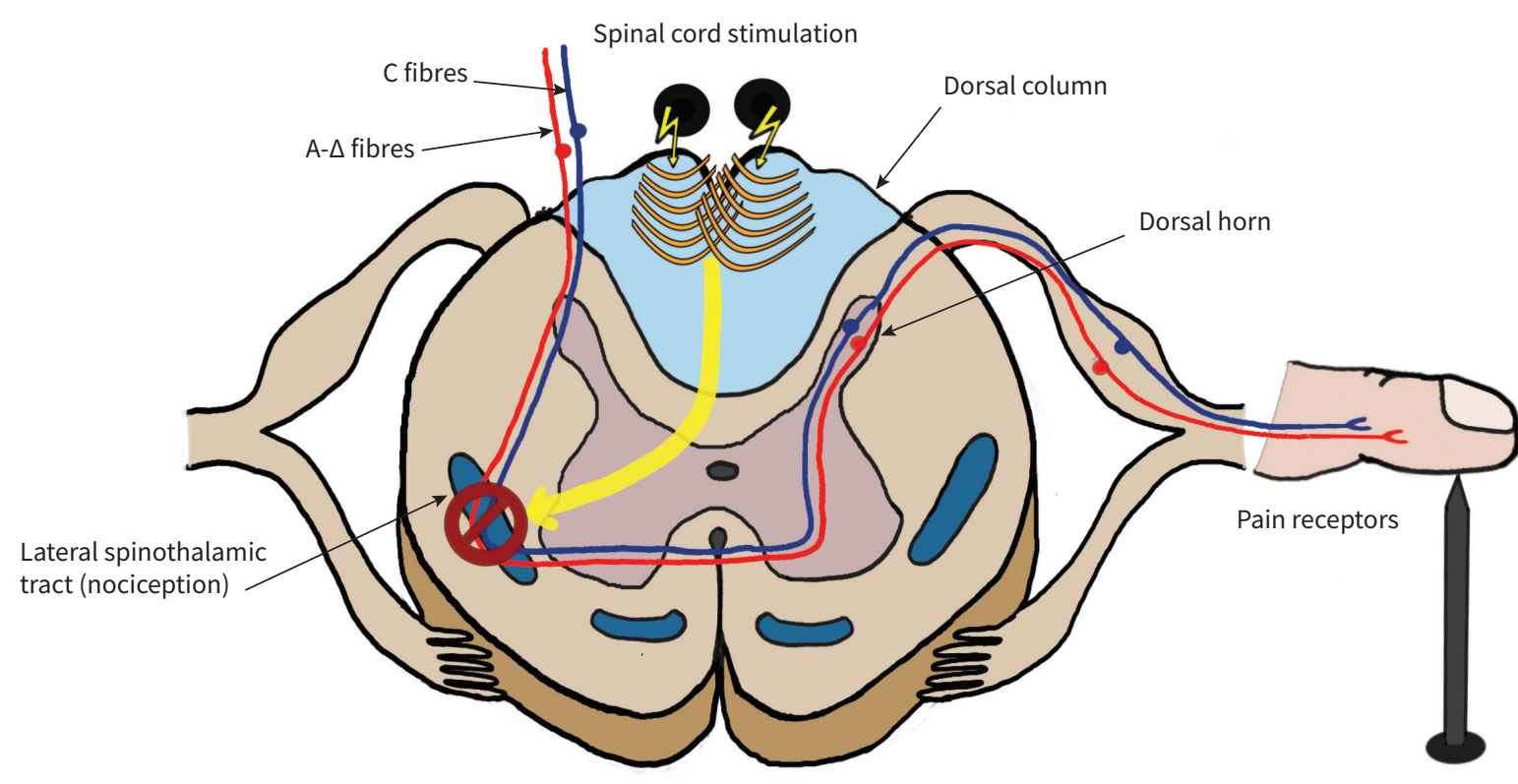

Spinal cord cross-section

Figure 1: Gate theory of pain. This theory postulates that pain signal transmission via the spinothalamic tract from pain-transmitting fibres (unmyelinated $\mathrm{C}$ fibres and lightly myelinated $\mathrm{A}-\Delta$ fibres) is blocked by stimulating the dorsal column large myelinated fibers and the polysynaptic interneurons (PSINs). The PSINs then fire back up on the dorsal horn, blocking pain receptors from synapsing. Illustration by Jean Chan.

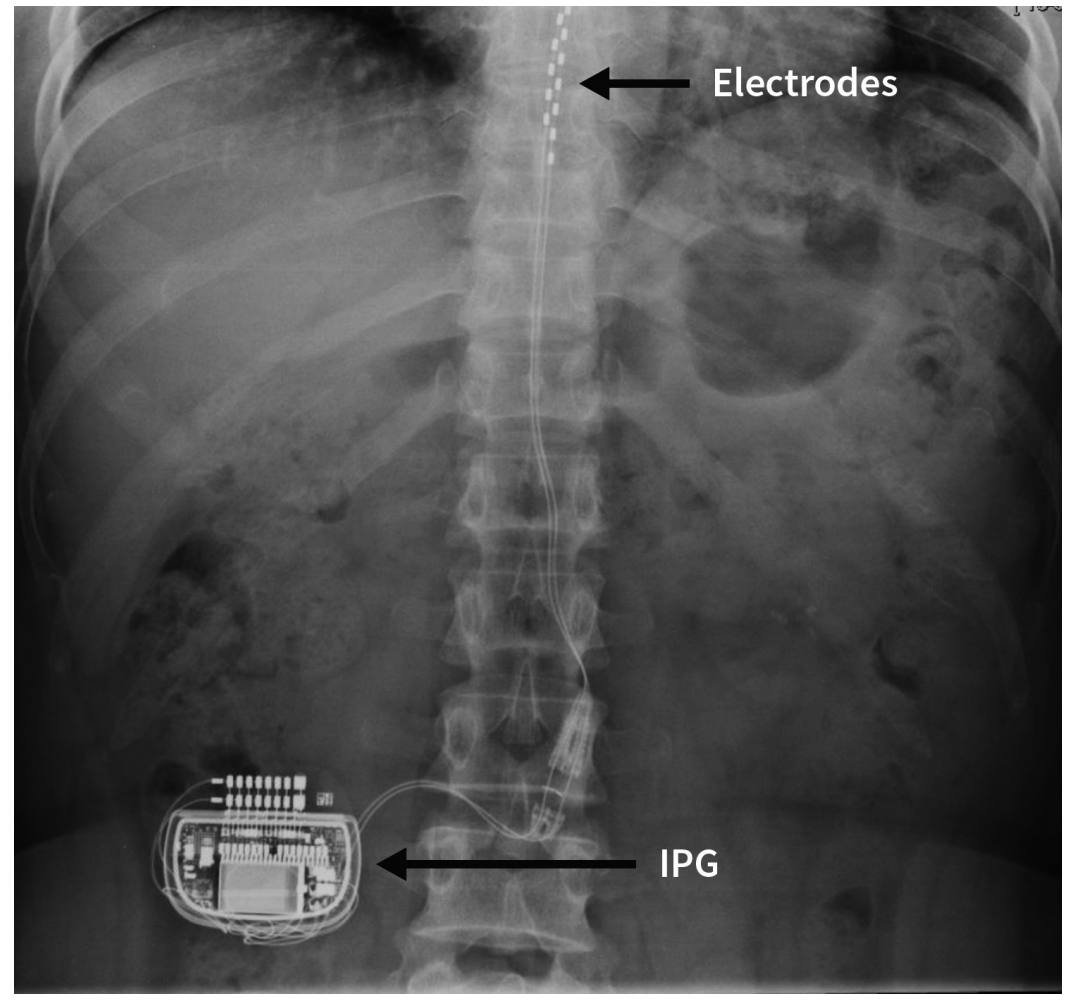

Figure 2: Radiograph of a spinal cord stimulator device. Note: IPG = implantable pulse generator.
Spinal cord stimulation is delivered in 2 phases: a trial phase and a permanent implant phase. Implantation of a trial system for about 1 week helps determine effectiveness. ${ }^{2}$ Only if $50 \%$ or greater improvement from baseline pain is achieved will permanent systems be implanted., ${ }^{2,7}$

Spinal cord stimulation referrals can be made to most Canadian university-based interventional pain specialists or spine surgeons.

\section{Who is eligible?}

Most chronic neuropathic pain conditions have been reported to respond to spinal cord stimulation, including failed back surgery syndrome, complex regional pain syndrome and chronic peripheral neuropathies. ${ }^{2,4,8}$ Additional expanding indications include refractory angina, diabetic neuralgia, post-herpetic neuralgia, and visceral or peripheral ischemic pain. ${ }^{2,4}$

Contraindications include active infections, immunosuppression and inability to withhold anticoagulation. ${ }^{2}$ People with a history of abuse or trauma, poor social support and substantial cognitive deficits tend to have poorer outcomes, and expert mental health assessment is recommended. ${ }^{2}$ 


\section{What are the potential harms?}

Spinal cord stimulation is generally safe because of its minimal invasiveness and reversibility. More common complications are hardware related, including electrode migration or fracture, and failure of the implantable pulse generator, but all are readily correctable. ${ }^{9}$ Biologic complications are less common, including seromas at the insertion site of the generator $(2.5 \%)$, local infections $(3.4 \%)$ and dural puncture headaches $(0.3 \%)$. Serious complications like epidural abscesses or hematomas and neurologic injuries are uncommon $(<0.3 \%){ }^{9}$

\section{What is the evidence?}

In a 2020 systematic review of 15 randomized controlled trials, Hofmeister and colleagues found a robust body of evidence supporting spinal cord stimulation. ${ }^{4}$ Below is a description of 3 of the most influential studies conducted on efficacy of the treatment.

The Patients with Failed Back Surgery Syndrome (PROCESS) study, conducted in 2007, was an international, multicentre, randomized trial comparing tonic spinal cord stimulation plus medical management, versus medical management alone in 100 patients with failed back surgery syndrome. The primary outcome was $50 \%$ or better leg pain relief on $10 \mathrm{~cm}$ visual analogue scale score (VAS). At 12 months, this was achieved in $34 \%$ of patients receiving spinal cord stimulation versus $7 \%$ of patients who were solely medically managed $(p=0.005)$. For secondary outcomes, patients receiving spinal cord stimulation experienced improved quality of life and functional capacity, with greater treatment satisfaction. Furthermore, 8 of 50 (16\%) patients receiving spinal cord stimulation ceased opioids, versus 1 of 44 (2\%) with sole medical management. ${ }^{6}$

The Senza study was a multicentre, randomized, pragmatic, parallel-arm, noninferiority trial comparing high-frequency spinal cord stimulation to traditional tonic stimulation in 198 patients with chronic intractable back and leg pain. The primary outcome was $50 \%$ or greater reduction on $10 \mathrm{~cm}$ VAS for back and leg pain without a stimulation-related neurologic deficit. At 12 months, high-frequency stimulation proved superior to tonic stimulation in reducing back and leg pain (79\% v. $51.3 \% ; p<0.001)$. Secondary outcomes included reduction or elimination of opioid consumption, which was higher in the high-frequency group (35.5\%) than in the tonic group $(26.4 \% ; p<0.05) .{ }^{10}$

The Success Using Neuromodulation With BURST (SUNBURST) trial compared burst with tonic stimulation in 100 patients. ${ }^{7}$ This multicentre, randomized, controlled crossover noninferiority trial found that burst stimulation was superior to tonic stimulation within each patient in overall improvement in $100 \mathrm{~mm}$ VAS. During the burst phase, $89 \%$ of patients experienced reduced to no paresthesia. After the initial crossover period and 1 year of followup, $68.2 \%$ preferred burst as compared with $23.9 \%$ who preferred tonic stimulation $(p<0.001){ }^{8}$

These trials were unblinded because the experience of paresthesia makes blinding infeasible. In the last 3-5 years, spinal cord stimulation technology has advanced, with "paresthesia-free" stimulus development. ${ }^{2}$ A subperception stimulus is produced using high-frequency spinal cord stimulation with $30 \mu \mathrm{s} 10 \mathrm{kHz}$ low-amplitude pulses, ${ }^{10}$ and using burst spinal cord stimulation emulating neuronal firing with stimuli bursts separated by a recovery phase. ${ }^{8}$ Both burst and high-frequency stimuli appear to be superior to traditional tonic stimulus. ${ }^{8,10}$ Paresthesia-free devices may allow for blinded studies in the future, and longerterm studies would help test efficacy of spinal cord stimulation beyond 2 years.

\section{Cost-effectiveness}

Chronic back and limb pain treatments are historically burdened with high upfront and recurrent costs. Hoelscher and colleagues ${ }^{7}$ reviewed cost data from 21 retrospective studies. Five studies performed cost-effectiveness analyses and found that results fell within usual third-party "willingness-to-pay" thresholds of US\$50000-US\$100000 quality-adjusted life-years gained. Information about long-term cost-effectiveness was limited mainly to modelling direct cost data, but durability of spinal cord stimulation treatment suggests that initial costs can be recovered within 2-3 years. ${ }^{7}$ There remains a need for longterm, prospective data from randomized trials that include both direct and indirect costs.

\section{What can be expected in the future?}

Recent Health Canada-approved neuromodulation devices allow for stimulation of the dorsal root ganglion rather than the spinal cord, which requires less energy and treats more targeted dermatomes. Some systems no longer require implantation of a pulse generator. Further refinement has also occurred by titrating stimulus strengths, combining stimulus types or even allowing stimulus titration based on body position (closed-loop spinal cord stimulator).

Improved battery technology is expected to make implantable pulse generators smaller, hold a greater charge or eliminate the need for an external induction charger. Remote communication may facilitate reprogramming from home, and wearable devices may allow parameter tracking, such as any increase in activity levels as a result of reduction in pain.

Currently, all provinces cover the cost of spinal cord stimulation but generally limit the number of device insertions each year. Data from randomized trials evaluating cost-effectiveness and the latest devices may further support the wider use of this novel technology.

\section{References}

1. Chronic pain in Canada: laying a foundation for action - a report by the Canadian Pain Task Force, June 2019. Ottawa: Health Canada; 2019.

2. Deer TR, Mekhail N, Provenzano D, et al.; Neuromodulation Appropriateness Consensus Committee. The appropriate use of neurostimulation of the spinal cord and peripheral nervous system for the treatment of chronic pain and ischemic diseases: the neuromodulation appropriateness consensus committee. Neuromodulation 2014;17:515-50, discussion 550.

3. Ontario Health (Quality). 10-kHz high-frequency spinal cord stimulation for adults with chronic noncancer pain: a health technology assessment. Ont Health Technol Assess Ser 2020;20:1-109. 
4. Hofmeister M, Memedovich A, Brown S, et al. Effectiveness of neurostimulation technologies for the management of chronic pain: a systematic review. Neuromodulation 2020;23:150-7.

5. Shealy CN, Mortimer JT, Reswick JB. Electrical inhibition of pain by stimulation of the dorsal columns: preliminary clinical report. Anesth Analg 1967;46:489-91.

6. Kumar K, Taylor RS, Jacques L, et al. Spinal cord stimulation versus conventional medical management for neuropathic pain: a multicentre randomised controlled trial in patients with failed back surgery syndrome. Pain 2007;132: 179-88.

7. Hoelscher C, Riley J, Wu C, et al. Cost-effectiveness data regarding spinal cord stimulation for low back pain. Spine 2017;42(Suppl 14):S72-9.

8. Deer T, Slavin KV, Amirdelfan K, et al. Success Using Neuromodulation With BURST (SUNBURST) study: results from a prospective, randomized controlled trial using a novel burst waveform. Neuromodulation 2018;21:56-66.

9. Deer TR, Mekhail N, Provenzano D, et al.; Neuromodulation Appropriateness Consensus Committee. The appropriate use of neurostimulation: avoidance and treatment of complications of neurostimulation therapies for the treatment of chronic pain. Neuromodulation 2014;17:571-97.

10. Kapural L, Cong Y, Doust M, et al. Novel $10-\mathrm{kHz}$ high-frequency therapy is superior to traditional low-frequency spinal cord stimulation for the treatment of chronic back and leg pain (SENZA-RCT). Anesthesiology 2015;123:851-60.
Competing interests: Aaron Hong reports receiving personal fees from Stimwave Canada and grants from Abbott Laboratories and Medtronic, outside the submitted work. No other competing interests were declared.

This article has been peer reviewed.

Affiliations: Department of Anesthesia (Hong, Hare, Mazer), St. Michael's Hospital; Department of Anesthesiology and Pain Medicine (Hong, Hare, Mazer), University of Toronto, Toronto, Ont.; Department of Anesthesia (Varshney), Providence Health Care, Vancouver, BC; Department of Physiology (Hare, Mazer), University of Toronto; Keenan Research Centre for Biomedical Science in the Li Ka Shing Knowledge Institute (Hare, Mazer), St. Michael's Hospital, Toronto, Ont.

Contributors: Aaron Hong and Vishal Varshney contributed to the conception and design of the work and drafted the manuscript. All of the authors contributed to the analysis and interpretation of data. All of the authors revised the manuscript critically for important intellectual content, gave final approval of the version to be published and agreed to be accountable for all aspects of the work.

Correspondence to: Aaron Hong, Aaron.Hong@unityhealth.to 